\title{
A Comment on Link Dynamics and Protocol Design in a Multi-Hop Mobile Environment
}

\author{
Abbas Nayebi \\ Research Institute of Petroleum Industry, West Blvd. of Azadi Sports Complex, Tehran, Iran \\ E-mail: nayebi@ripi.ir \\ Received 22 January, 2011; revised 27 January, 2011; accepted 15 March, 2011
}

\begin{abstract}
This paper is a supplementary document for "Link Dynamics and Protocol Design in a Multi-Hop Mobile Environment" [1], which presents an analytic model to obtain link lifetime in mobile wireless networks. The model outcome is used as groundwork for several mobility-related evaluations. Here, it is discussed how an improper assumption in the probabilistic model led to some errors in the resultant expressions. After a detailed description of the erratic assumption, a modification is proposed to correct the derivations. The results of the new model are verified by the simulation results provided at the original paper.
\end{abstract}

Keywords: Link Lifetime, Probabilistic Models

\section{Background}

This paper is intended to supplement Samar and Wicker's paper Link Dynamics and Protocol Design in a Multi-Hop Mobile Environment [1]. Therefore, the redundant proofs are avoided here and the reader is referred to the original document. Paper [1] (and also the earlier version [2]) is basically an analytic paper that aims to provide a model for average link lifetime and the distribution of link lifetime in wireless networks. Besides, the paper presents derivations for new link arrival/change rate and new link arrival/change time distribution. Finally, the authors conducted some simulation experiments and tried to validate their analytic results by the experimental ones. At the first glance, the simulation results for the expected link lifetime deviates considerably (Figure 1), which is justified in the paper by the simulation errors. However, the simulation results for expected new link arrival rate and new link inter-arrival time PDF tightly match the analytic ones (Figure 2) without a notable simulation error (the same simulation settings are used). Moreover, in the results, link lifetime PDF (probability density function) is provided only for a limited case of $v_{1}=0$. As we will prove later in the text, this is the only case that hides the errors in the derivations of the PDF. First of all, we need to confirm that the analytic results of the model are incorrect. To do so, we apply a straightforward, high level, and simple test to verify that the simulation result in Figure $\mathbf{1}$ is correct

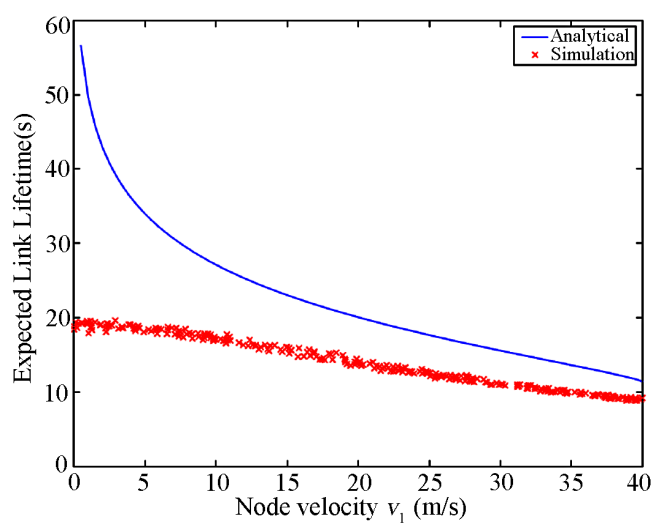

(a)

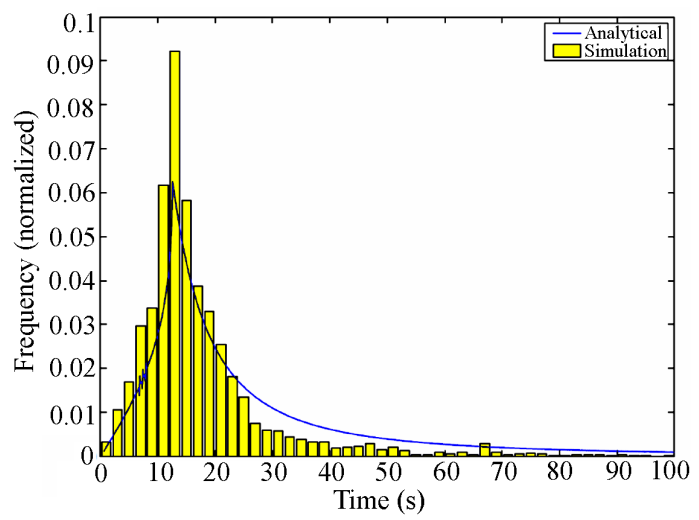

(b)

Figure 1. Comparison with simulation statistics: (a) Expected link lifetime. (b) Link Lifetime PDF for a node with velocity $v_{1}=0 \mathrm{~m} / \mathrm{s}$ (from [1]). 


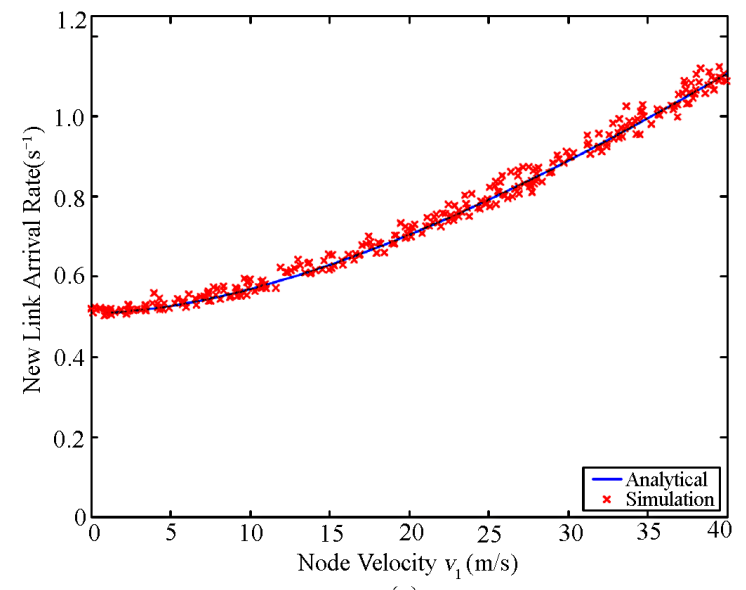

(a)

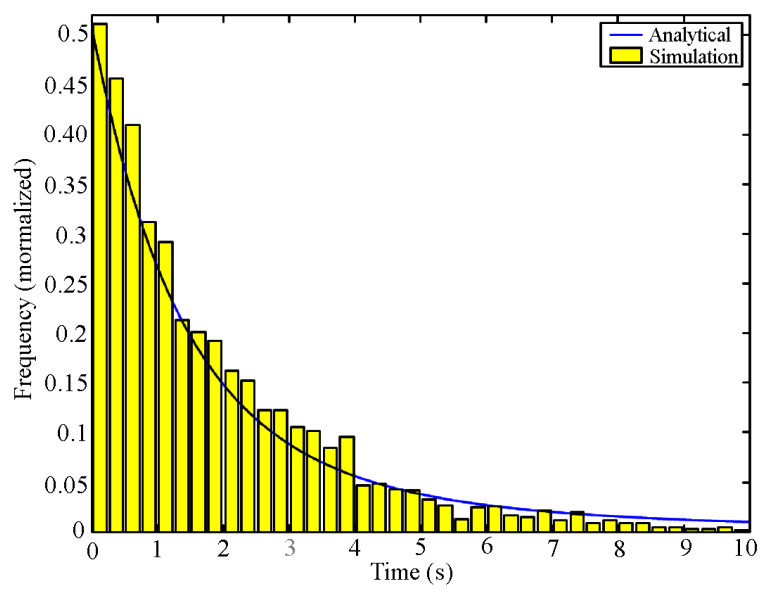

(b)

Figure 2. Comparison with simulation statistics: (a) Expected new link arrival rate. (b) New link interarrival time PDF for a node with velocity $v_{1}=0 \mathrm{~m} / \mathrm{s}$ (from [1])

and the analytic one is incorrect. According to the Little's theorem, the average number of customers in a system is equal to the average arrival rate multiplied by the average time each customer spends in the system. In analogy, here we have: the average number of neighbors in transmission area of a node is equal to the average new link arrival rate multiplied by the expected link lifetime. The simulation settings for the results in Figure 1 are adjusted to have 10 nodes in average in transmission area of each node. For an arbitrary node velocity of $v_{1}=$ $10 \mathrm{~m} / \mathrm{s}$ the results in Figure 2 gives average arrival rate of $0.57 \mathrm{~s}^{-1}$. Thus, according to the Little's theorem, for this velocity the correct expected link lifetime is about $10 / 0.57=17.54$ seconds which matches the simulation curve in Figure 1, while the analytic curve gives 27.5 seconds for $v_{1}=10 \mathrm{~m} / \mathrm{s}$. In the rest of the text, the erroneous assumptions in the derivations that results in this error are described.

\section{Error in derivations of the average link lifetime, new link inter-arrival time, and link breakage inter-arrival time}

As a brief review, the authors used $v_{1}, v_{2}, v, \varphi, \alpha$ and $\theta$ to denote the velocity vectors of node 1 and 2 , the magnitude of the relative velocity, the angle that relative velocity vector makes with $\boldsymbol{v}_{1}$, the angle that shows the arrival point of node 2 into the transmission area of node 1 , and the angle between $\boldsymbol{v}_{1}$ and $\boldsymbol{v}_{2}$, respectively. The authors used the joint PDF of $v, \varphi$ and $\alpha$, i.e. $f_{v \varphi \alpha}($.$) to extract the average link lifetime (Equation (9)$ in [1]) and distribution of link lifetime (Equation (14) in [1]).

Actually, the problem is with assumptions used in the derivation of $f_{v \varphi \alpha}($.$) . In evaluation of the link lifetime$ using $f_{v \rho \alpha}($.$) , it is assumed that:$

- $v$ is the magnitude of relative velocity of a node that is entering the transmission range of $v_{1}$, but in the evaluation of $f_{v \varphi \alpha}($.$) in the appendix it is assumed that:$

- $v$ is the relative velocity of a uniformly selected random node over the network.

This slight difference in the assumptions led to the errors in the evaluations. Please note that the velocity distribution of a randomly selected node over the network differs from the velocity distribution of a node entering the transmission area of a moving node because nodes with larger relative velocities are more likely to be visited. To clarify the problem further, let's consider a simplified one dimensional model (say, a street) in which half of the nodes move towards right and the other half move towards left both with velocity $2 \mathrm{~m} / \mathrm{s}$. Suppose that a particular node 1 is moving with velocity $1 \mathrm{~m} / \mathrm{s}$ towards right. The relative velocities with the two categories of nodes moving right and left are $1 \mathrm{~m} / \mathrm{s}$ and $3 \mathrm{~m} / \mathrm{s}$, respectively. The corresponding $f_{v \varphi}($.$) as evaluated in$ the appendix of [1] provides probability of $1 / 2$ for the both values of relative velocities. However, node 1 visits the nodes moving towards left three times more frequent than the nodes moving towards right in average. Thus, the probability that a visited node has relative velocity of $1 \mathrm{~m} / \mathrm{s}$ and $3 \mathrm{~m} / \mathrm{s}$ are $1 / 4$ and $3 / 4$, respectively, and not $1 / 2$ for each of them. In other words, each moving node tends to visit other nodes with higher magnitude of relative velocity more often and it does not visit all the other nodes with different relative velocities with the same rate. Thus, the average magnitude of relative velocity between node 1 and a visited node is higher than the average magnitude of relative velocity between node 1 and a randomly selected node over the network. That is why the analytically obtained average link lifetime (Figure 1) is higher than the actual one (the simulation curve). To 
see the point that this assumption is considered in the model, refer to the text after Expression (51) in [1], which is " $v_{2}$ is uniformly distributed between a and $b$. Also, from Assumption 3, $\theta$ is uniformly distributed between 0 and $2 \pi$ ". As mentioned above, these assumptions are true only for a randomly selected node of the network not for the nodes that visit a particular moving node.

With the same justification, the derivations of new link inter-arrival time (Expression (25) in [1]) and link breakage inter-arrival time (Expression (30) in [1]) are also incorrect. Please note that only in a particular case that node 1 is fixed the results are correct since relative velocity is equal to absolute velocity. As the simulation results for the link lifetime and the inter-arrival distributions (Figure 11-ii, 12-ii, and 13-ii in [1]) are provided only for this particular case, they do not depict the errors.

Here, one may ask that why the results for the average rate of new arrivals is correct while it is also based on the same $f_{v \varphi}($.$) function calculated in the appendix. In order$ to answer this question the reader may refer to Figure 4 in [1] and Expression (18) there. The average number of nodes with velocity $v_{\mathbf{1}}$ entering the zone within the next $t$ seconds is reported as $2 R \sigma v t \cdot f(v, \varphi) \mathrm{d} v \mathrm{~d} \varphi$. In this Expression there is a factor $v$ (relative velocity). Thus, in this case, the nodes with higher magnitudes of relative velocity are considered with higher rate of arrival and the analytic results are correct. Using the same correction, other results of the paper can be corrected. To do so, instead of using $f_{v \varphi \alpha}($.$) obtained in the appendix of [1],$ we need to use a new pdf weighted with the relative velocity ( $v)$ as

$$
\frac{f_{v \phi \alpha}(v, \phi, \alpha) v}{\int_{-\pi}^{\pi} \int_{-\pi}^{\pi} \int_{0}^{\infty} f_{v \phi \alpha}(v, \phi, \alpha) v \mathrm{~d} v \mathrm{~d} \phi \mathrm{d} \alpha}
$$

The model is re-implemented and the new PDF is applied to the model. Figure 3(a) compares the result for expected link lifetime obtained by applying the new PDF and the results with the previous PDF. Comparison of the figure with Figure 3(b) (original figure) shows the new analytic curve tightly matches the simulation curve. Thus, the error in the original figure was not due to simulation error and it was due to the wrong probabilistic assumption. Moreover, the figure validates the correctness of the corrected model.

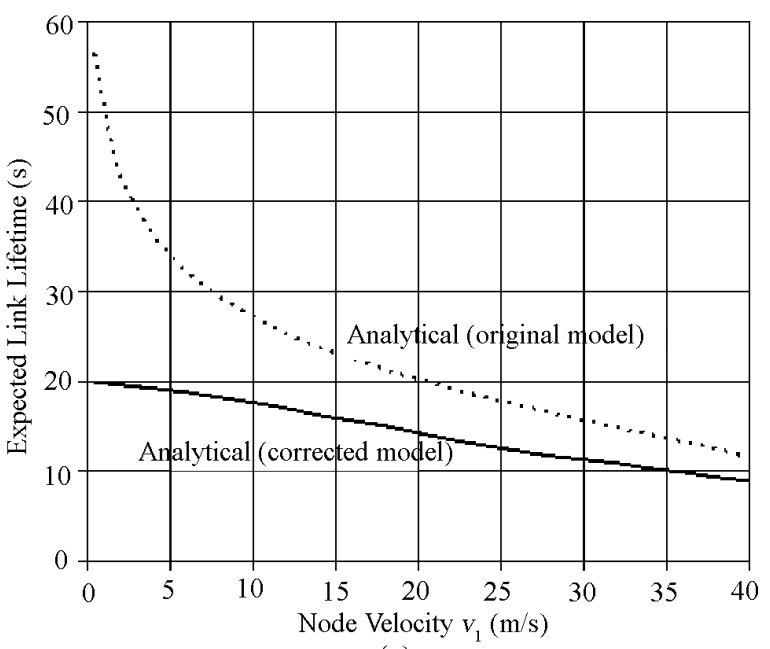

(a)

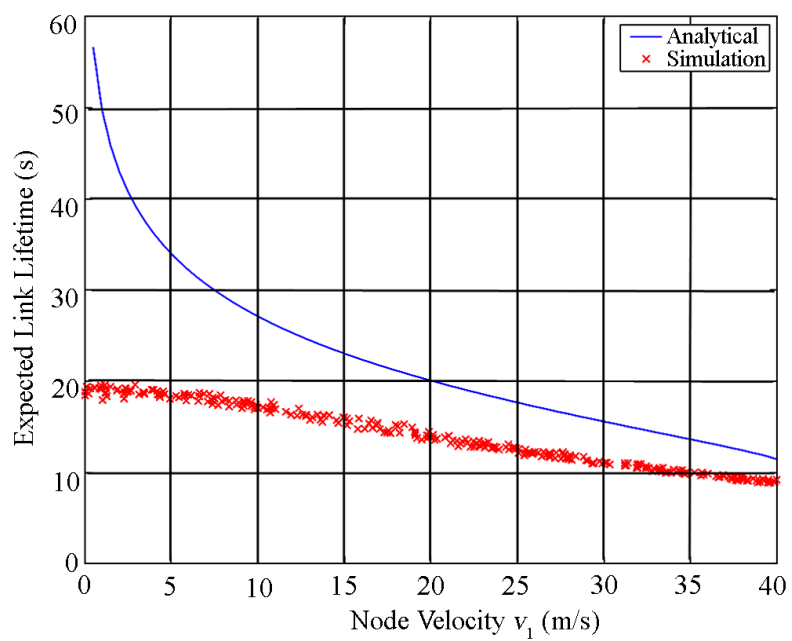

(b)

Figure 3. (a): Analytical expected link lifetime vs. node velocity obtained from the original model and the corrected model. (b): Analytical and simulation results from [1] (gridlines are added for comparison). The simulation results is matched with the corrected analytical model.

\section{References}

[1] P. Samar and S. B. Wicker, "Link Dynamics and Protocol Design in a Multihop Mobile Environment," IEEE Transactions on Mobile Computing, Vol. 5, No. 9, 2006, pp. 1156-1172. doi:10.1109/TMC.2006.131

[2] P. Samar and S. B. Wicker, "On the Behavior of Communication Links of a Node in a Multi-Hop Mobile Environment," Proceedings of the Fifth ACM International on Mobile Ad Hoc Networking and Computing (MobiHoc), Roppongi, 2004, pp. 145-156. 